\title{
On Efficiency of First and Second Order
}

\author{
P. J. Bickel ${ }^{1}$, D. M. Chibisov ${ }^{2}$ and W. R. van Zwet ${ }^{3}$ \\ Department of Statistics, University of California, Berkeley, U.S.A., Steklov Mathematical \\ Institute, Moscow, U.S.S.R. and Department of Mathematics, University of Leiden, \\ The Netherlands
}

\section{Summary}

It has been noted by a number of authors that if two tests are asymptotically efficient for the same testing problem, then typically their powers will not only agree to first but also to second order. A general result of this type was given by Pfanzagl (1979) in a paper entitled 'First order efficiency implies second order efficiency'. Because of their technical nature, however, these contributions give little insight into the nature of this phenomenon. The purpose of the present paper is to provide an intuitive understanding of the phenomenon by proving a simple theorem of this kind under mild assumptions.

Key words: Asymptotic efficiency of tests; Second order efficiency; Deficiency.

\section{Introduction}

For $N=1,2, \ldots$, we consider an experiment with outcome $X_{N}$ taking values in an arbitrary sample space. Let $P_{N, 0}$ and $P_{N, 1}$ be two possible distributions of $X_{N}$, with densities $p_{N, 0}$ and $p_{N, 1}$ with respect to some dominating measure $\mu_{N}$. We shall write $E_{N, 0}$ and $E_{N, 1}$ for expectations under $P_{N, 0}$ and $P_{N, 1}$ respectively. Define the logarithm of the likelihood ratio by

$$
\Lambda_{N}=\log \frac{p_{N, 1}\left(X_{N}\right)}{p_{N, 0}\left(X_{N}\right)}
$$

with the usual conventions for vanishing $p_{N, 0}$ and/or $p_{N, 1}$.

Now consider a sequence $\alpha_{N} \in(0,1)$ and let $\phi_{N}\left(\Lambda_{N}, \alpha_{N}\right)$ denote the test function of the most powerful level- $\alpha_{N}$ test for $P_{N, 0}$ against $P_{N, 1}$; thus

$$
\phi_{N}\left(\Lambda_{N}, \alpha_{N}\right)= \begin{cases}0 & \text { for } \Lambda_{N}<c_{N}\left(\alpha_{N}\right) \\ 1 & \text { for } \Lambda_{N}>c_{N}\left(\alpha_{N}\right)\end{cases}
$$

with

$$
E_{N, 0} \phi_{N}\left(\Lambda_{N}, \alpha_{N}\right)=\alpha_{N}, \quad E_{N, 1} \phi_{N}\left(\Lambda_{N}, \alpha_{N}\right)=\pi_{N}^{*}\left(\alpha_{N}\right),
$$

where $\pi_{N}^{*}\left(\alpha_{N}\right)$ is the maximum attainable power against $P_{N, 1}$ at level $\alpha_{N}$.

For $N=1,2, \ldots$, let $Z_{N}$ be a random variable depending only on the outcome $X_{N}$ of the $N$ th experiment and let $\psi_{N}\left(Z_{N}, \alpha_{N}\right)$ denote the test function of the level- $\alpha_{N}$ right-sided test based on the statistic $Z_{N}$, i.e.

$$
\psi_{N}\left(Z_{N}, \alpha_{N}\right)= \begin{cases}0 & \text { for } Z_{N}<d_{N}\left(\alpha_{N}\right) \\ 1 & \text { for } Z_{N}>d_{N}\left(\alpha_{N}\right)\end{cases}
$$

\footnotetext{
${ }^{1}$ Research supported by the U.S. Office of Naval Research, Contract N00014-80-C-0163, the National Science Foundation, Grant MC S76 10238 A01, and by the Netherlands' Organization for Pure Scientific Research.

${ }^{2}$ Research supported by the Netherlands' Organization for Pure Scientific Research

${ }^{3}$ Research supported by the U.S. Office of Naval Research, Contract N00014-80-C-0163.
} 
We have

$$
E_{N, 0} \psi_{N}\left(Z_{N}, \alpha_{N}\right)=\alpha_{N}, \quad E_{N, 1} \psi_{N}\left(Z_{N}, \alpha_{N}\right)=\pi_{N}\left(\alpha_{N}\right),
$$

where $\pi_{N}\left(\alpha_{N}\right)$ is the power of this test against $P_{N, 1}$.

For a sequence $\tau_{N} \in(0,1]$, we shall say that the sequence of level- $\alpha_{N}$ tests $\psi_{N}\left(Z_{N}, \alpha_{N}\right)$ is $\tau_{N}-$ efficient if, for $N \rightarrow \infty$,

$$
\pi_{N}^{*}\left(\alpha_{N}\right)-\pi_{N}\left(\alpha_{N}\right)=o\left(\tau_{N}\right)
$$

In a more usual terminology first and second order efficiency correspond to $\tau_{N}$-efficiency with $\tau_{N}=1$ and $\tau_{N}=N^{-1 / 2}$ respectively.

Finally, let us define for $N=1,2, \ldots$,

$$
\Delta_{N}= \begin{cases}0 & \text { if } Z_{N}=\Lambda_{N}= \pm \infty, \\ Z_{N}-\Lambda_{N} & \text { otherwise, }\end{cases}
$$

and let us denote the indicator function of a set $B$ by $1_{B}$.

Having established our notation, we now give an informal description of the phenomenon we wish to study. Let us think of $N$ as denoting sample size, i.e. $N$ is the number of independent random variables involved in the $N$ th testing problem. We are interested primarily in sequences of testing problems where $\alpha_{N} \geq \varepsilon$ and $\pi_{N}^{*}\left(\alpha_{N}\right) \leq 1-\varepsilon$ for some $\varepsilon>0$ and all $N$. Such sequences exist if it is impossible to discriminate perfectly between $P_{N, 0}$ and $P_{N, 1}$ even as $N \rightarrow \infty$ and this is true if $P_{N, 0}$ and $P_{N, 1}$ are contiguous. A sufficient condition for contiguity, and one which is often fulfilled in this case, is asymptotic normality of $\Lambda_{N}$ both under $P_{N, 0}$ and $P_{N, 1}$. But if $\Lambda_{N}$ is asymptotically normal, it will usually also be possible to obtain an Edgeworth expansion for its distribution function under $P_{N, 0}$ and $P_{N, 1}$ and this will yield a similar expansion for the power of the test based on $\Lambda_{N}$, viz.

$$
\pi_{N}^{*}\left(\alpha_{N}\right)=c_{0}+c_{1} N^{-1 / 2}+o\left(N^{-1 / 2}\right) .
$$

Typically the remainder term on the right in (1.1) will be $O\left(N^{-1}\right)$.

Suppose that the sequence of tests $\psi_{N}\left(Z_{N}, \alpha_{N}\right)$ is asymptotically efficient to first order, or 1-efficient in our terminology. For most statistical problems such 1-efficient tests abound. They are usually based on statistics $Z_{N}$ that closely resemble $\Lambda_{N}$. Typically $\Delta_{N}=Z_{N}-\Lambda_{N}$ tends to zero in probability both under $P_{N, 0}$ and $P_{N, 1}$ and in the situation we have described so far, this suffices to ensure 1-efficiency. Of course, these 1-efficient tests can also be based on statistics $Z_{N}$ which do not resemble $\Lambda_{N}$ at all, because the test statistic associated with a test is by no means unique. However, we shall not be concerned with such alternative representations and suppose that $\Delta_{N} \rightarrow 0$ in $P_{N, 0^{-}}$and in $P_{N, 1}$-probability.

Recalling that $N$ is the sample size, we note that one often finds that a sequence of random variables $\Delta_{N}$ tending to zero in probability does so at the rate of $N^{-1 / 2}$. Thus, for 1-efficient tests, $N^{1 / 2} \Delta_{N}$ will typically be bounded in probability both under $P_{N, 0}$ and $P_{N, 1}$. Hence, one may expect to be able to establish Edgeworth expansions for the distribution functions of $Z_{N}$ under $P_{N, 0}$ and $P_{N, 1}$, which differ from those for $\Lambda_{N}$ only in the term of order $N^{-1 / 2}$ and in those of smaller order. This yields a similar expansion for the power of the test based on $Z_{N}$,

$$
\pi_{N}\left(\alpha_{N}\right)=c_{0}+c_{1}^{\prime} N^{-1 / 2}+o\left(N^{-1 / 2}\right),
$$

where the remainder term on the right will typically be $O\left(N^{-1}\right)$ or of slightly larger order. The fact that the leading terms in expansions (1.1) amd (1.2) have the same value $c_{0}$ reflects the 1-efficiency of the sequence $\psi_{N}\left(Z_{N}, \alpha_{N}\right)$. There would seem to be no reason a priori to expect that also $c_{1}=c_{1}^{\prime}$, which would entail $N^{-1 / 2}$-efficiency or efficiency of second order.

However, in those cases where expansions (1.1) and (1.2) were explicitly computed, one does indeed find that $c_{1}=c_{1}^{\prime}$ and hence that the sequence $\psi_{N}\left(Z_{N}, \alpha_{N}\right)$ is $N^{-1 / 2}$-efficient. This 
phenomenon was noticed by Pfanzagl (1973), (1975) and Chibisov (1974) for a number of tests for the parametric one-sample problem and by Bickel \& van Zwet (1978) for rank tests for the nonparametric two-sample problem. Some tests for the one-sample problem for the case where nuisance parameters are present were considered by Chibisov (1973) and Pfanzagl (1974) and also found to be $N^{-1 / 2}$-efficient. Finally, it was shown by Pfanzagl (1979) that first-order efficiency forces second-order efficiency for a large class of one-sample tests in the presence of nuisance parameters. With an appropriate definition of efficiency a similar result was obtained for estimators.

In each of these contributions, $N^{-1 / 2}$-efficiency is established by imposing the conditions needed to obtain expansions (1.1) and (1.2) and then checking that these expansions are in fact identical. This method of proof coupled with its extreme technicality makes an intuitive understanding of the phenomenon rather difficult. The purpose of the present paper is to provide such an intuitive understanding by proving a simple theorem of this kind under rather mild assumptions. Since our aim is to provide insight rather than generality, we shall only be concerned with the simple hypothesis testing problem described above and avoid the technicalities inherent in the treatment of nuisance parameters and estimation problems, although extension to these situations is certainly possible. Having mentioned estimation, however, we should note that Rao's $(1961,1962)$ concept of second order efficiency of estimators as discussed by Efron (1975) and Ghosh, Sinha \& Wieand (1980), refers to optimality up to $o\left(N^{-1}\right)$ and would therefore correspond to $N^{-1}$-efficiency or third order efficiency in our terminology. This difference in terminology is not as illogical as it may seem because most results of these authors concern the performance of an estimator as measured by its risk relative to a symmetric loss function and expansions for this quantity typically do not contain a term of order $N^{-1 / 2}$, so that the term of order $N^{-1}$ is indeed the second order term in this case.

In section 2 we present our result, discuss its meaning and explain why it is true. A formal proof of the theorem is given in section 3. Though this proof is straightforward, the nonmathematically inclined may wish to skip it.

\section{Discussion of the result}

We adopt the notation and conventions introduced in the previous section. In particular we recall that $\tau_{N}$ is an arbitrary sequence in $(0,1]$ and that first and second order efficiency correspond to $\tau_{N}$-efficiency with $\tau_{N}=1$ and $\tau_{N}=N^{-1 / 2}$ respectively.

\section{THEOREM. SUPpose that}

$$
\underset{N}{\lim \inf } \alpha_{N}>0
$$

and that there exists $A>0$ such that for every $x_{0} \in \mathbb{R}$, every $\gamma>0$ and $N \rightarrow \infty$,

$$
\begin{aligned}
& \sup _{x \leq x_{0}} P_{N, 0}\left(x-\tau_{N}^{1 / 2} \leq \Lambda_{N} \leq x\right)=O\left(\tau_{N}^{1 / 2}\right), \\
& E_{N, 0}\left|\Delta_{N}\right| 1_{\left(\gamma \tau_{N}^{1 / 2}, A\right)}\left(\left|\Delta_{N}\right|\right)=o\left(\tau_{N}\right), \\
& P_{N, 0}\left(\Delta_{N} \geq A\right)=o\left(\tau_{N}\right), \\
& P_{N, 1}\left(\Delta_{N} \leq-A\right)=o\left(\tau_{N}\right) .
\end{aligned}
$$

Then the sequence of tests $\psi_{N}\left(Z_{N}, \alpha_{N}\right)$ is $\tau_{N}$-efficient.

Let us briefly discuss the conditions of this theorem. First, assumption (2.2) is clearly satisfied for any sequence $\tau_{N}$ if the distributions of $\Lambda_{N}$ under $P_{N, 0}$ possess uniformly bounded densities. 
More generally, (2.2) will hold if the distribution functions $F_{N}$ of $\Lambda_{N}$ under $P_{N, 0}$ can be approximated with a uniform error of order $\tau_{N}^{1 / 2}$ by distribution functions $G_{N}$ with uniformly bounded densities, i.e. if $\sup \left|F_{N}(x)-G_{N}(x)\right|=O\left(\tau_{N}^{1 / 2}\right)$. This is certainly the case when $F_{N}$ has a normal approximation or an Edgeworth expansion with the required error.

If $\tau_{N} \rightarrow 0$, assumption (2.2) clearly implies that the distributions of $\Lambda_{N}$ under $P_{N, 0}$ do not tend to a degenerate limit. In view of this, conditions (2.3)-(2.4) serve to ensure that under $P_{N, 0},\left|\Delta_{N}\right|$ is small compared to the variation of $\Lambda_{N}$. Note that these conditions refer only to values of $\left|\Delta_{N}\right|$ which excede $\gamma \tau_{N}^{1 / 2}$ and that they are satisfied if the distribution of $\Delta_{N}$ under $P_{N, 0}$ either assigns probability 1 to a set where $\left|\Delta_{N}\right|=o\left(\tau_{N}^{1 / 2}\right)$, or has at most very small tails outside that range. Thus, roughly speaking, $\Delta_{N}$ is required to be $o\left(\tau_{N}^{1 / 2}\right)$ under $P_{N, 0}$; under $P_{N, 1}$ condition (2.5) is even weaker, but this is to a certain extent artificial and is due to our efforts to replace conditions under $P_{N, 1}$ as much as possible by conditions under $P_{N, 0}$ which will usually be easier to verify. It follows that one cannot hope to say more about the differences between the distribution functions of $\Lambda_{N}$ and $Z_{N}$ under $P_{N, 0}$ and $P_{N, 1}$ than that they are $o\left(\tau_{N}^{1 / 2}\right)$. One would therefore expect to be able to prove that $\pi_{N}^{*}\left(\alpha_{N}\right)-\pi_{N}\left(\alpha_{N}\right)=o\left(\tau_{N}^{1 / 2}\right)$ but, somewhat surprisingly, the conclusion of the theorem is the stronger statement that $\pi_{N}^{*}\left(\alpha_{N}\right)-\pi_{N}\left(\alpha_{N}\right)=$ $o\left(\tau_{N}\right)$. The condition that roughly $\Delta_{N}$ is $o\left(\tau_{N}^{1 / 2}\right)$ under $P_{N, 0}$ cannot essentially be improved. By taking $\Delta_{N}=\Delta \tau_{N}^{1 / 2}$ where $\Delta$ is independent of $\Lambda_{N}$ for every $N$, one easily constructs examples where $\pi^{*}\left(\alpha_{N}\right)-\pi\left(\alpha_{N}\right)$ is of exact order $\tau_{N}$.

A formal proof of the theorem will be given in section 3. At this point we shall be content to provide an intuitive explanation of the result by sketching the proof for the special case where there exist numbers $\delta_{N}$ such that for $N=1,2, \ldots$,

$$
\left|\Delta_{N}\right|<\delta_{N}=o\left(\tau_{N}^{1 / 2}\right)
$$

We should perhaps stress that a boundedness assumption like (2.6) is not likely to be fulfilled in concrete examples. It is made here merely to avoid technicalities at this stage and bring out the essential simplicity of the proof.

Let us write $c_{N}$ and $d_{N}$ for $c_{N}\left(\alpha_{N}\right)$ and $d_{N}\left(\alpha_{N}\right)$ respectively. Since the tests $\phi_{N}\left(\Lambda_{N}, \alpha_{N}\right)$ and $\psi_{N}\left(Z_{N}, \alpha_{N}\right)$ have the same level $\alpha_{N},(2.6)$ clearly implies that we may assume that $\left|c_{N}-d_{N}\right|<\delta_{N}$. Invoking (2.6) once more, we see that if $\Lambda_{N} \geq c_{N}$ and $Z_{N} \leq d_{N}$, then $d_{N}-\delta_{N} \leq \Lambda_{N} \leq d_{N}+\delta_{N}$; the same conclusion holds if $\Lambda_{N} \leq c_{N}$ and $Z_{N} \geq d_{N}$. It follows that on the set where $\phi_{N}\left(\Lambda_{N}, \alpha_{N}\right) \neq$ $\psi_{N}\left(Z_{N}, \alpha_{N}\right)$ we have $\left|\Lambda_{N}-d_{N}\right| \leq \delta_{N}$, and again because both tests have level $\alpha_{N}$ we find with the aid of (2.2),

$$
\begin{aligned}
\pi_{N}^{*}\left(\alpha_{N}\right)-\pi_{N}\left(\alpha_{N}\right) & =E_{N, 1}\left\{\phi_{N}\left(\Lambda_{N}, \alpha_{N}\right)-\psi_{N}\left(Z_{N}, \alpha_{N}\right)\right\} \\
& =E_{N, 0}\left(e^{\Lambda_{N}}-e^{d_{N}}\right)\left\{\phi_{N}\left(\Lambda_{N}, \alpha_{N}\right)-\psi_{N}\left(Z_{N}, \alpha_{N}\right)\right\} \\
& \leq e^{d_{N}}\left(e^{\delta_{N}}-1\right) P_{N, 0}\left(\left|\Lambda_{N}-d_{N}\right| \leq \delta_{N}\right) \\
& =O\left(\delta_{N} \tau_{N}^{1 / 2}\right)=o\left(\tau_{N}\right) .
\end{aligned}
$$

Note that we need to have $d_{N}$ bounded above, but this is an easy consequence of (2.1) and (2.6). The above sketch should make it clear that the essential thing which makes the theorem work is that not only do $\phi_{N}\left(\Lambda_{N}, \alpha_{N}\right)$ and $\psi_{N}\left(Z_{N}, \alpha_{N}\right)$ resemble each other closely, but that also $\Lambda_{N}$ is almost constant on the set where they differ.

Let us finally discuss the relevance of the theorem to the problem of first and second order efficiency. As was pointed out in section 1 , first order efficiency of $\psi_{N}\left(Z_{N}, \alpha_{N}\right)$ will typically imply that $N^{1 / 2} \Delta_{N}$ is bounded in probability both under $P_{N, 0}$ and $P_{N, 1}$. Also, $E_{N, 0}\left|N^{1 / 2} \Delta_{N}\right|^{r}$ and $E_{N, 1}\left|N^{1 / 2} \Delta_{N}\right|^{r}$ will usually be bounded for some $r>1$ and this (or even uniform integrability of $\left|N^{1 / 2} \Delta_{N}\right|$ under $P_{N, 0}$ and $\left.P_{N, 1}\right)$ is amply sufficient to ensure that assumptions (2.3)-(2.5) are satisfied for $\tau_{N}=N^{-1 / 2}$. But then the theorem ensures that $\psi_{N}\left(Z_{N}, \alpha_{N}\right)$ is efficient to second order under the very mild conditions (2.1) and (2.2) for $\tau_{N}=N^{-1 / 2}$. 
An examination of our proof shows that if we replace (2.6) by

$$
\left|\Delta_{N}\right| \leq M \tau_{N}^{1 / 2}
$$

for $M<\infty$ and $N=1,2, \ldots$, then we obtain the conclusion

$$
\pi_{N}^{*}\left(\alpha_{N}\right)-\pi_{N}\left(\alpha_{N}\right)=O\left(\tau_{N}\right)
$$

Taking $\tau_{N}=N^{-1}$ we conclude that if $\left|N^{1 / 2} \Delta_{N}\right|$ is bounded and if, e.g., the distribution of $\Lambda_{N}$ under $P_{N, 0}$ tends to normality at the rate of $N^{-1 / 2}$, then $\pi_{N}^{*}\left(\alpha_{N}\right)-\pi_{N}\left(\alpha_{N}\right)=O\left(N^{-1}\right)$. This means that the tests based on $Z_{N}$ have a finite deficiency in the sense of Hodges \& Lehmann (1970). That is, if we let $\alpha_{N}=\alpha \in(0,1)$ for $N=1,2, \ldots$ and define $N^{\prime}$ to be the smallest integer for which $\pi_{N^{\prime}}(\alpha) \geq \pi_{N}^{*}(\alpha)$ then $\lim \sup \left(N^{\prime}-N\right)<\infty$.

Assumption (2.7) is of course not necessary to obtain (2.8) and $N^{-1 / 2}$-efficiency is frequently coupled with finite deficiency. However, the coupling is not inevitable. An example, in a nuisance parameter context, is provided by the normal scores test studied by Bickel \& van Zwet (1978). Note that this bears out what we have said about the order of the remainder terms in (1.1) and (1.2).

\section{Proof of the theorem}

Take a sequence $\alpha_{N} \in(0,1)$ satisfying $(2.1)$ and write $c_{N}=c_{N}\left(\alpha_{N}\right)$ and $d_{N}=d_{N}\left(\alpha_{N}\right)$. If $d_{N}=-\infty$ for some $N$ then

$$
\begin{aligned}
1-\pi_{N}\left(\alpha_{N}\right) & \leq P_{N, 1}\left(Z_{N}=-\infty\right) \leq P_{N, 1}\left(\Lambda_{N}=-\infty\right)+P_{N, 1}\left(\Delta_{N}=-\infty\right) \\
& =P_{N, 1}\left(\Delta_{N}=-\infty\right)=o\left(\tau_{N}\right)
\end{aligned}
$$

because of (2.5), and $\psi_{N}\left(Z_{N}, \alpha_{N}\right)$ is clearly $\tau_{N}$-efficient. Take $A$ as in (2.3)-(2.5). Then

$$
\begin{aligned}
\alpha_{N} & \leq P_{N, 0}\left(Z_{N} \geq d_{N}\right) \leq P_{N, 0}\left(\Lambda_{N} \geq d_{N}-A\right)+P_{N, 0}\left(\Delta_{N} \geq A\right) \\
& \leq e^{-d_{N}+A} P_{N, 1}\left(\Lambda_{N} \geq d_{N}-A\right)+o\left(\tau_{N}\right) \leq e^{-d_{N}+A}+o\left(\tau_{N}\right)
\end{aligned}
$$

because of (2.4). In view of (2.1) it is therefore no loss of generality to assume that for some $D<\infty$ and all $N$,

$$
-\infty<d_{N} \leq D
$$

Define

$$
\bar{\Delta}_{N}= \begin{cases}d_{N}-\Lambda_{N} & \text { if } \Lambda_{N}<d_{N} \leq Z_{N} \text { or } Z_{N} \leq d_{N}<\Lambda_{N} \\ 0 & \text { otherwise. }\end{cases}
$$

Obviously,

$$
\bar{\Delta}_{N}^{+} \leq \Delta_{N}^{+}, \bar{\Delta}_{N}^{-} \leq \Delta_{N}^{-}
$$

where $x^{+}=x \vee 0$ and $x^{-}=(-x) \vee 0$ denote the positive and negative parts of a number $x$.

Let $\alpha_{N}^{\prime}$ be such that $d_{N}=c_{N}\left(\alpha_{N}^{\prime}\right)$. Then

$$
\begin{aligned}
\left\{\pi_{N}^{*}\left(\alpha_{N}\right)-\pi_{N}\left(\alpha_{N}\right)\right\}+\left\{\pi_{N}^{*}\left(\alpha_{N}^{\prime}\right)-\pi_{N}^{*}\right. & \left.\left(\alpha_{N}\right)-e^{d_{N}}\left(\alpha_{N}^{\prime}-\alpha_{N}\right)\right\} \\
& =\pi_{N}^{*}\left(\alpha_{N}^{\prime}\right)-\pi_{N}\left(\alpha_{N}\right)-e^{d_{N}}\left(\alpha_{N}^{\prime}-\alpha_{N}\right) \\
& =E_{N, 0}\left\{\phi_{N}\left(\Lambda_{N}, \alpha_{N}^{\prime}\right)-\psi_{N}\left(Z_{N}, \alpha_{N}\right)\right\}\left(e^{\Lambda_{N}}-e^{d_{N}}\right) \\
& =E_{N, 0}\left|\phi_{N}\left(\Lambda_{N}, \alpha_{N}^{\prime}\right)-\psi_{N}\left(Z_{N}, \alpha_{N}\right)\right| \mid e^{\Lambda_{N}}-E^{d_{N} \mid} \\
& \leq e^{d_{N}} E_{N, 0}\left|e^{-\Delta_{N}}-1\right|,
\end{aligned}
$$


since $\left\{\phi_{N}\left(\Lambda_{N}, \alpha_{N}^{\prime}\right)-\psi_{N}\left(Z_{N}, \alpha_{N}\right)\right\}$ is nonnegative, or nonpositive, if $\left(\Lambda_{N}-d_{N}\right)$ is positive, or negative, and equals zero if $\bar{\Delta}_{N}=0$ and $\left(\Lambda_{N}-d_{N}\right) \neq 0$. A similar argument yields

$$
\pi_{N}^{*}\left(\alpha_{N}^{\prime}\right)-\pi_{N}^{*}\left(\alpha_{N}\right)-e^{d_{N}}\left(\alpha_{N}^{\prime}-\alpha_{N}\right)=E_{N, 0}\left\{\phi_{N}\left(\Lambda_{N}, \alpha_{N}^{\prime}\right)-\phi_{N}\left(\Lambda_{N}, \alpha_{N}\right)\right\}\left(e^{\Lambda_{N}}-e^{d_{N}}\right) \geq 0
$$

and hence

$$
0 \leq \pi_{N}^{*}\left(\alpha_{N}\right)-\pi_{N}\left(\alpha_{N}\right) \leq e^{d_{N}} E_{N, 0}\left|e^{-\bar{\Delta}_{N}}-1\right| .
$$

By (2.3) there exists a sequence $\gamma_{N} \downarrow 0$ such that

$$
E_{N, 0}\left|e^{-\Delta_{N}}-1\right| 1_{\left(\gamma_{N} \tau_{N}^{1 / 2}, A\right)}\left(\left|\Delta_{N}\right|\right)=o\left(\tau_{N}\right)
$$

for $N \rightarrow \infty$. In view of (3.2) this implies

$$
E_{N, 0}\left|e^{-\bar{\Delta}_{N}}-1\right| 1_{\left(\gamma_{N} \tau_{N}^{1 / 2}, \infty\right]}\left(\left|\bar{\Delta}_{N}\right|\right) 1_{[0, A)}\left(\left|\Delta_{N}\right|\right)=o\left(\tau_{N}\right) .
$$

Also (3.1) and (2.2) with $x_{0}=D+1$ yield

$$
\begin{aligned}
E_{N, 0}\left|e^{-\bar{\Delta}_{N}}-1\right| 1_{\left|0, \gamma_{N} \tau_{N}^{1 / 2}\right|}\left(\left|\bar{\Delta}_{N}\right|\right) & =O\left(\gamma_{N} \tau_{N}^{1 / 2} P_{N, 0}\left(0<\left|\bar{\Delta}_{N}\right| \leq \gamma_{N} \tau_{N}^{1 / 2}\right)\right) \\
& =o\left(\tau_{N}^{1 / 2} P_{N, 0}\left(0<\left|\Lambda_{N}-d_{N}\right| \leq \tau_{N}^{1 / 2}\right)\right)=o\left(\tau_{N}\right) .
\end{aligned}
$$

If $\Delta_{N} \geq 0$ then $\bar{\Delta}_{N} \geq 0$ and because of (2.4)

$$
E_{N, 0}\left|e^{-\bar{\Delta}_{N}}-1\right| 1_{[A, \infty]}\left(\Delta_{N}\right) \leq P_{N, 0}\left(\Delta_{N} \geq A\right)=o\left(\tau_{N}\right) .
$$

If $\Delta_{N} \leq 0$ then $\bar{\Delta}_{N} \leq 0$ and (2.5) ensures that

$$
\begin{aligned}
e^{d_{N}} E_{N, 0}\left|e^{-\bar{\Delta}_{N}}-1\right| 1_{[-\infty,-A]}\left(\Delta_{N}\right) & \leq e^{d_{N}} E_{N, 0} e^{-\bar{\Delta}_{N}} 1_{[-\infty,-A]}\left(\Delta_{N}\right) 1_{[-\infty, 0)}\left(\bar{\Delta}_{N}\right) \\
& \leq E_{N, 0} e^{\Lambda_{N}} 1_{[-\infty, A]}\left(\Delta_{N}\right)=P_{N, 1}\left(\Delta_{N} \leq-A\right)=o\left(\tau_{N}\right) .
\end{aligned}
$$

Together (3.1) and (3.3)-(3.7) imply that $\pi_{N}^{*}\left(\alpha_{N}\right)-\pi_{N}\left(\alpha_{N}\right)=o\left(\tau_{N}\right)$ and the proof is complete.

\section{References}

Bickel, P.J. \& van Zwet, W.R. (1978). Asymptotic expansions for the power of distribution free tests in the two-sample problem. Ann. Statist. 6, 937-1004.

Chibisov, D.M. (1973). Asymptotic expansions for Neyman's $C(\alpha)$ tests. Proc. Second Japan-USSR Symp. on Probability Theory, ed. G. Maruyama and Yu. V. Prokhorov. Lecture Notes in Mathematics 330, pp. 16-45. Springer, Berlin.

Chibisov, D.M. (1974). Asymptotic expansions for some asymptotically optimal tests. Proc. Prague Symp. on Asymptotic Statistics, ed. J. Hájek, 2, 37-68.

Efron, B. (1975). Defining the curvature of a statistical problem (with applications to second order efficiency). Ann. Statist. 3, 1189-1242.

Ghosh, J.K., Sinha, B.K. \& Wieand, H.S. (1980). Second order efficiency of the MLE with respect to a bounded bowl-shaped loss function. Ann. Statist. 8, 506-521.

Hodges, J.L. \& Lehmann, E.L. (1970). Deficiency. Ann. Math. Statist. 41, 783-801.

Pfanzagl, J. (1973). Asymptotic expansions related to minimum contrast estimators. Ann. Statist. 1, 993-1026.

Pfanzagl, J. (1974). Asymptotically optimum estimation and test procedures. Proc. Prague Symp. on Asymptotic Statistics, ed. J. Hájek, 1, 201-272.

Pfanzagl, J. (1975). On asymptotically complete classes. Statistical Inference and Related Topics, 2, ed. M. L. Puri, pp. 1-43. Academic Press, New York.

Pfanzagl, J. (1979). First order efficiency implies second order efficiency. Contributions to Statistics [J. Hájek Memorial Volume], ed. J. Jurečková, pp. 167-196. Academia, Prague,

Rao, C.R. (1961). Asymptotic efficiency and limiting information. Proc. 4th Berkeley Symp. 1, 531-545.

Rao, C.R. (1962). Efficient estimates and optimum inference procedures in large samples (with discussion). $J . R$. Statist. Soc. B 24, 46-72. 


\section{Résumé}

Plusieurs auteurs ont remarqué, que, si deux tests sont asymptotiquement efficients pour le même problème de test statistique, leurs puissances s'accorderont normalement non seulement du premier mais aussi du deuxième ordre. Pfanzagl (1979) donna un résultat général de ce genre dans son article "First order efficiency implies second order efficiency". Cependant, à cause de leur structure technique, ces contributions ne donnent qu'une idée peu claire de la nature de ce phénomène. Le but de cet article-ci est d'établir une notion intuïtive du phénomène en démontrant un théorème simple de ce genre sous des conditions souples.

[Paper received October 1980, revised January 1981] 